\title{
d2Dplot: 2D X-ray diffraction data processing and analysis for through-the-substrate microdiffraction
}

\section{Oriol Vallcorba and Jordi Rius}

J. Appl. Cryst. (2019). 52, 478-484

\section{IUCr Journals CRYSTALLOGRAPHY JOURNALS ONLINE}

Copyright (C) International Union of Crystallography

Author(s) of this article may load this reprint on their own web site or institutional repository provided that this cover page is retained. Republication of this article or its storage in electronic databases other than as specified above is not permitted without prior permission in writing from the IUCr.

For further information see http://journals.iucr.org/services/authorrights.html 
JOURNAL OF

APPLIED

CRYSTALLOGRAPHY

ISSN 1600-5767

Received 12 November 2018

Accepted 10 February 2019

Edited by A. Barty, DESY, Hamburg, Germany

Keywords: 2D X-ray diffraction; through-thesubstrate microdiffraction; powder diffraction; computer programs.

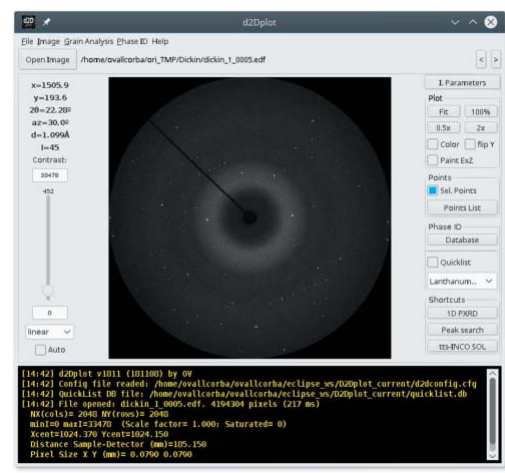

(C) 2019 International Union of Crystallography

\section{d2Dplot: 2D X-ray diffraction data processing and analysis for through-the-substrate microdiffraction}

\author{
Oriol Vallcorba ${ }^{\mathrm{a} *}$ and Jordi Rius ${ }^{\mathrm{b}}$ \\ ${ }^{\mathbf{a}}$ ALBA Synchrotron Light Source, Cerdanyola del Vallès, Barcelona, Spain, and ${ }^{\mathbf{b}}$ Institut de Ciència de Materials de \\ Barcelona (ICMAB-CSIC), Cerdanyola del Vallès, Spain. *Correspondence e-mail: ovallcorba@cells.es
}

The $d 2$ Dplot computer program provides a set of tools for the visualization, processing and analysis of $2 \mathrm{D}$ X-ray diffraction (2DXRD) data. Among the operations available there are the sum/subtraction of 2DXRD images, conversion to 1D data (powder pattern), azimuthal plotting, calibration of instrumental parameters, background subtraction and a command-line mode to run operations inside data processing pipelines. The graphical user interface allows easy use of the program. It also includes two main features: (i) the possibility of creating a user compound database to help in the fast phase identification of similar samples, and (ii) a detailed peak analysis routine for the application of the through-the-substrate microdiffraction methodology.

\section{Introduction}

The collection of diffraction data using $2 \mathrm{D}$ detectors is nowadays common practice. Depending on the nature of the sample and the purpose of the experiment, different data processing procedures are applied (He, 2009). For polycrystalline samples, data measured as $2 \mathrm{D} \mathrm{X}$-ray diffraction (2DXRD) images are often integrated to generate the corresponding 1D powder diffraction patterns. For single crystals, 2DXRD data are routinely collected with the rotation method using small steps (Arndt \& Wonacott, 1977) and then processed to obtain the list of measured reflections with their respective intensities. Sometimes, however, it can be important to inspect the individual images for specific sample features like texture or graininess. There are several software solutions available for processing 2DXRD patterns, such as the well-established and complete FIT2D (Hammersley, 1997, 2016) and the general-purpose GSAS-II (Toby \& Von Dreele, 2013). Also, software for specific data processing is available, such as XDS (Kabsch, 1988), CrysAlis (Agilent Technologies, 2014), DIALS (Winter et al., 2018) and MOSFLM (Battye et $a l .$, 2011) for single-crystal data reduction, $a d x v$ (Arvai, 1994) for the visualization of images, and libraries for azimuthal integration of 2DXRD images such as pyFAI (Kieffer \& Karkoulis, 2013; Ashiotis et al., 2015).

The computer program $d 2 \mathrm{Dplot}$ has been designed to prioritize the visual inspection of 2DXRD images while also implementing basic data processing operations such as the summation or subtraction of frames, conversion of 2DXRD data to 1D data, azimuthal plotting, calibration of instrumental parameters, background subtraction and a commandline mode to run operations inside data processing pipelines. It also includes two main features: (i) the possibility of creating a limited database with a reduced number of compounds (those most frequently occurring) to help in the fast phase identification of similar samples, and (ii) a detailed peak analysis 
routine whose output can be used either independently or as input for the application of the through-the-substrate microdiffraction methodology (tts- $\mu$ XRD) described by Rius et al. (2015). This methodology allows analysis of transmission diffraction data from polished thin sections mounted on glass substrates and has already been applied to the solution of petrological/mineralogical, cultural heritage and metallurgical problems (Vallcorba et al., 2017; Maritan, Piovesan et al., 2018; Schmalen et al., 2018). Analysis of tts- $\mu$ XRD data is the main motivation for $d 2 D$ plot. It was created as the graphical tool for the development of the tts- $\mu$ XRD methodology and now it has become a key part of its application, providing the peak search and integration algorithms and helping during the selection of the correct crystal orientation(s). $d 2$ Dplot has also proved useful in sample mapping by tts- $\mu$ XRD, offering a quick identification of phases during the selection of the zones to be measured.

The most relevant aspects of $d 2$ Dplot will be described in the next sections.

\section{Technical description, availability and requirements}

d2Dplot has been written in Java and can be executed on any PC with the Java runtime environment installed (version 1.6.0_10 or higher). The following third-party libraries have been used: Apache Commons Math (version 3.6.1, Apache licence), MigLayout (version 4.3, written by Mikael Grev, BSD licence) and imageJ [version 1.50i (Schneider et al., 2012), Public Domain] for reading and writing images in TIFF format. The software can be downloaded from the ALBA Synchrotron Light Source web site (https://www.cells.es/en/ beamlines/bl04-mspd/preparing-your-experiment) and includes a user manual detailing the options and operations that can be performed as well as the file formats that can be used. The

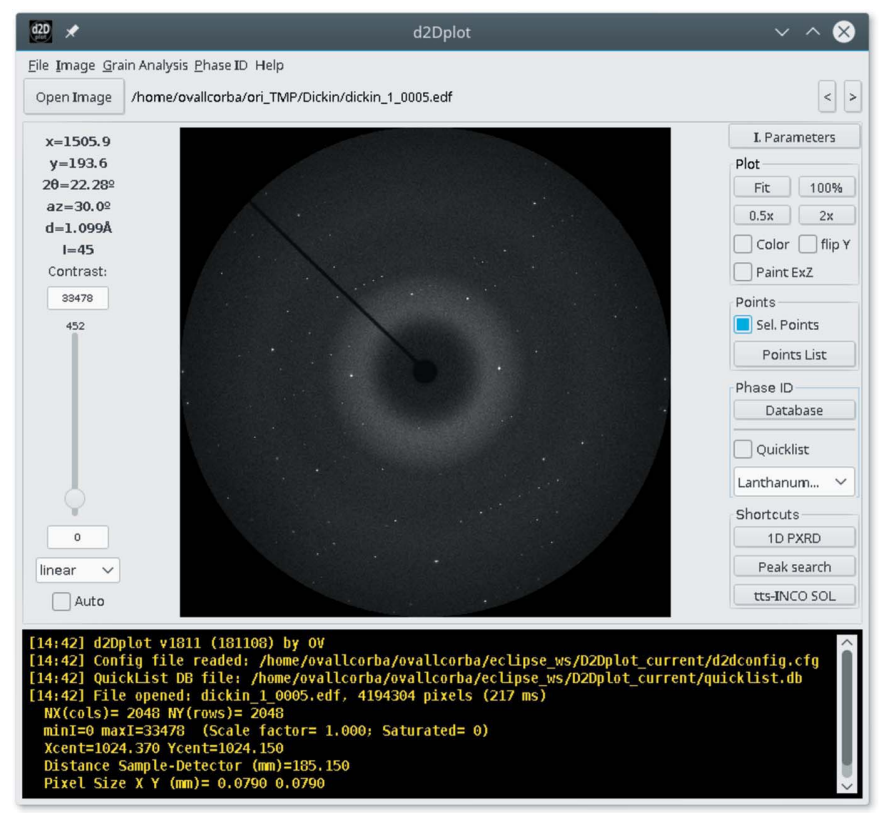

Figure 1

Main window of $d 2 D$ plot after opening a 2DXRD frame. software can be used free of charge for non-commercial and academic purposes.

\section{Program functionality and features}

The main window of the program (Fig. 1) contains (i) a top menu allowing access to all the options and operations it can perform, (ii) a left panel with information about the current pixel of the image pointed to by the mouse and the image contrast adjustment, (iii) a right panel with shortcuts to the most common operations, (iv) a bottom log panel with output messages from the program and $(\mathrm{v})$ the central region displaying the current opened frame. The interaction with the opened frame is done by using the mouse (left, right, middle buttons and the wheel). Several 2DXRD data formats are supported by $d 2$ Dplot, namely EDF (ESRF data format, http:// www.esrf.eu/computing/scientific/SAXS/doc/SaxsKeywords/ SaxsKeywords.pdf), IMG (Arvai \& Nielsen, 1983), GFRM (Bruker, 1999), SPR, CBF v1 (Bernstein \& Hammersley, 2005) and TIFF. If present, the instrumental information within the image header is read. This information can be accessed and edited inside the 'Image Parameters' dialog. If consecutive frames with the same name are numbered, easy change of images is possible by simply clicking the next/previous buttons.

\subsection{Basic 2DXRD processing}

d2Dplot provides a set of processing tools to prepare the 2DXRD data for further analysis. The main operations available are (i) summation or subtraction of frames (pixel-bypixel operations), (ii) conversion between data formats, (iii) calibration of instrumental parameters, (iv) definition of excluded zones, (v) background subtraction, (vi) conversion to 1D powder diffraction pattern, and (vii) generation of azimuthal plots. Details of the most relevant operations are listed below.

3.1.1. Calibration of instrumental parameters. Preliminary estimates. The experimental sample-to-detector distance $(D)$, the beam centre position on the detector plane (given by the $x_{\mathrm{o}}$ and $y_{\mathrm{o}}$ pixels) and the orthogonality of the detector during the experiment are derived from the $2 \mathrm{D}$ powder diffraction data of a calibrant substance. The program includes $\mathrm{LaB}_{6}$ and $\mathrm{Si}$, but other substances can be added as calibrants by introducing a list of $d$ spacings in the configuration file. The detector orthogonality deviation is defined by two angles: (i) the tilt angle measuring the deviation from the orthogonality of the beam with respect to the detector plane, and (ii) the rot angle describing the clockwise rotation from the vertical $y$ axis to the minor axis of the ellipse (fitted to the experimental Debye ring) as shown in Fig. 2. The definition of the detector deviation from orthogonality using two orthogonal angles is the most extended method to correct this distortion in the calculation of the effective $2 \theta$ for each pixel (Hinrichsen et al., 2008; Hart et al., 2013), and is the method normally used in diffraction software, e.g. FIT2D (Hammersley et al., 1994).

The calibration is carried out by taking the values of the detector distance, the wavelength and the beam centre from the frame header (image parameters) and by automatically 


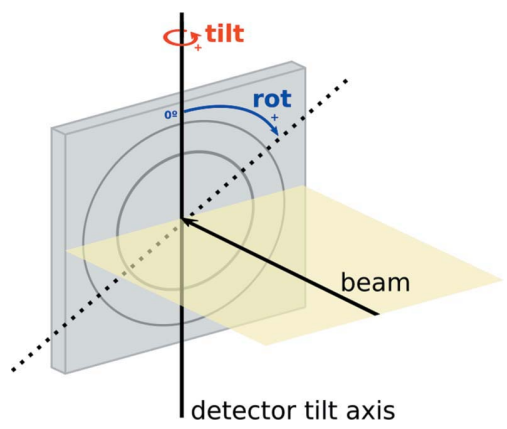

Figure 2

tilt and rot angles used to define the deviation from detector orthogonality with respect to the beam.

selecting or, alternatively, by clicking several points on the elliptically shaped inner Debye ring of the calibrant. After the least-squares ellipse fitting to the Debye ring, the estimates of the instrumental parameters, $\left(x_{\mathrm{o}}, y_{\mathrm{o}}\right), D$, tilt and rot, are obtained from the expressions (Hart et al., 2013)

$$
\begin{gathered}
\text { tilt }=-\sin ^{-1}(e \cos 2 \theta), \\
D=\frac{R_{\mathrm{m}}^{2}}{R_{\mathrm{M}} \tan 2 \theta}, \\
c=R_{\mathrm{M}} \tan 2 \theta \tan t i l t,
\end{gathered}
$$

where $R_{\mathrm{M}}, R_{\mathrm{m}}$ and $e$ are the major axis, minor axis and eccentricity of the ellipse, respectively. The beam position on the detector is calculated from $c$, which is the distance from the ellipse centre to the beam position on the detector surface along the major axis. This process is automatically repeated from the inner to the outer Debye rings, thereby using the new estimates of the instrumental parameters. As preliminary estimates of the instrumental parameters, the mean values over all the rings are taken.

Refinement of the instrumental parameters. By starting with the preliminary instrumental parameters, the refined ones are obtained by minimization of the sum of absolute differences between the theoretical and experimental $2 \theta$ values of the calibrant reflections,

$$
\sum_{i} \sum_{j(i)}\left|2 \theta_{i}-2 \theta_{j}\right|
$$

where the outer sum extends over the considered calibrant rings and the inner sum over the $\left(x_{j}, y_{j}\right)$ points on each calculated $i$ ellipse. Following the description by Hinrichsen $e t$ al. (2008), the calculated $2 \theta$ for a given measured pixel $(x, y)$, defined from the beam centre at $\left(x_{\mathrm{o}}, y_{\mathrm{o}}\right)$ and considering the detector tilt and rot as defined in $d 2$ Dplot, is

$$
2 \theta=\tan ^{-1}\left[\frac{x_{R o}^{2} \cos ^{2} t i l t+y_{R o}^{2}}{\left(D / \cos t i l t+x_{R o} \sin t i l t\right)^{2}}\right]^{1 / 2}
$$

wherein $\left(x_{R o}, y_{R o}\right)$ results from applying the $(-r o t)$ rotation to $(x, y)$, namely $x_{R o}=x \cos r o t-y \sin$ rot,$\quad y_{R o}=x \sin r o t+$ $y \cos$ rot.

3.1.2. Excluded zones. The frame zones excluded from further calculations can be set by an intensity threshold, a frame margin, a detector radius, using geometrical shapes (polygon, arc, beamstop) or painting the pixels with the mouse. The information on the excluded zones can be saved either as an independent text file or as a zero-valued mask image with excluded pixel intensities equal to -1 .

3.1.3. Background subtraction. Background subtraction is normally necessary when the intensity contribution from the holder (e.g. glass) is significant. A background image can be directly subtracted from the data or, alternatively, the background intensity at a given pixel can be estimated by averaging either square areas (avsq, with the side in pixels as the $N$ parameter) or arc-shaped areas (avarc, with the radial width and azimuthal aperture as parameters) around the pixel in several iterations. This is equivalent to the smoothing procedure described by Brückner (2000) adapted to 2D data.

For non-polycrystalline data there are two additional faster but less accurate procedures available: (i) the circular averaging of the pixel intensities in the corresponding $2 \theta$ ellipse (avarc); (ii) the selection of the minimum value among I0, I1, $\mathrm{I} 2$ and I3, where I0 is the intensity of the pixel and I1, I 2 and I3 are the intensities of the related pixels resulting from the application of a mirror reflection to the image on the $x$ axis, $y$ axis and both, respectively. Instead of single pixels, square zones (minsq) or arc-shaped zones (minarc) are used.

3.1.4. Conversion of a powder 2DXRD image to a 1D powder diffraction pattern. The $1 \mathrm{D}$ powder diffraction pattern is obtained from the 2DXRD image by populating a histogram at regular $2 \theta$ intervals with the pixel intensities and posterior division by the number of pixels contributing to each interval (the excluded pixels are not taken into account). The $2 \theta$ of each pixel is calculated with expression (5). The minimum interval size allowed is the smallest $2 \theta$ value variation between two adjacent pixels. Azimuthal bins (or 'cakes') can also be defined. For randomly oriented powders, the Lorentz and polarization factors affecting the intensities of the 1D pattern (Lipson et al., 2004) are, respectively,

$$
\begin{gathered}
L=\frac{\sin ^{2} 2 \theta}{\cos \theta}, \\
P=\frac{1}{2}\left(1+\cos ^{2} 2 \theta\right) .
\end{gathered}
$$

Since powder patterns taken on conventional diffractometers have the same Lorentz-polarization expression, direct comparison between them is possible. The conversion to a $1 \mathrm{D}$ powder pattern is useful not only for phase identification purposes, for example in combination with the PDF4 file of the International Centre for Diffraction Data (Faber \& Fawcett, 2002), but also for Rietveld refinement or for quantitative analysis.

Even for tts- $\mu$ XRD experiments with significant substrate contribution (e.g. glass support), the conversion to a $1 \mathrm{D}$ pattern is helpful. Recent applications are the characterization of laser-welded Al-Cu joints (Schmalen et al., 2018) and the identification of secondary phases in ancient ceramics (Maritan, Casas et al., 2018).

3.1.5. Azimuthal (circular) plot. By selecting this operation the pixel intensity evolution along an ellipse specified by the 
$2 \theta$ angle and a given tolerance ( $2 \theta$ window in degrees) is plotted. This plot allows extraction of valuable information on the grain coarseness and/or sample texture.

\subsection{Grain analysis}

d2D plot includes a set of tools to process 2DXRD images containing contributions from a single or several grains. This is one of its main features which also complements the tts$\mu \mathrm{XRD}$ methodology for the analysis of transmission diffraction data from thin sections mounted on glass substrates (Rius et al., 2015). tts- $\mu$ XRD consists of a series of measurements at selected sample points by using the rotation method with wide angular overlapping increments (typically between 5 and $15^{\circ}$ ) to cope with eventual diffracting volume variations along the section depth during the measurements (Rius, Vallcorba, Crespi \& Colombo, 2017). The orientation of the crystal(s) is then found, and the intensities are extracted and scaled by using all the measurements. The integrated intensities can be used later for structure solution and/or refinement. $d 2 \mathrm{Dplot}$ assists tts- $\mu \mathrm{XRD}$ by finding the spots on the image, extracting the intensities, preparing the input files, launching the tts_software (Rius, Vallcorba \& Frontera, 2017) and, finally, checking the orientation results at the various stages (Fig. 3). The program modules and the main options involved in these steps are detailed below.

3.2.1. Peak identification module. To find the peaks on the image, the search algorithm first applies several tests to each image pixel. These check the following:

(1) That the pixel intensity is greater than a threshold. The threshold is set as $I_{\text {mean }}+n \sigma_{I}$, where $I_{\text {mean }}$ is the mean intensity of the image and $\sigma_{I}$ is the corresponding standard deviation. A default value of $n$ can be set by the program. If the back-

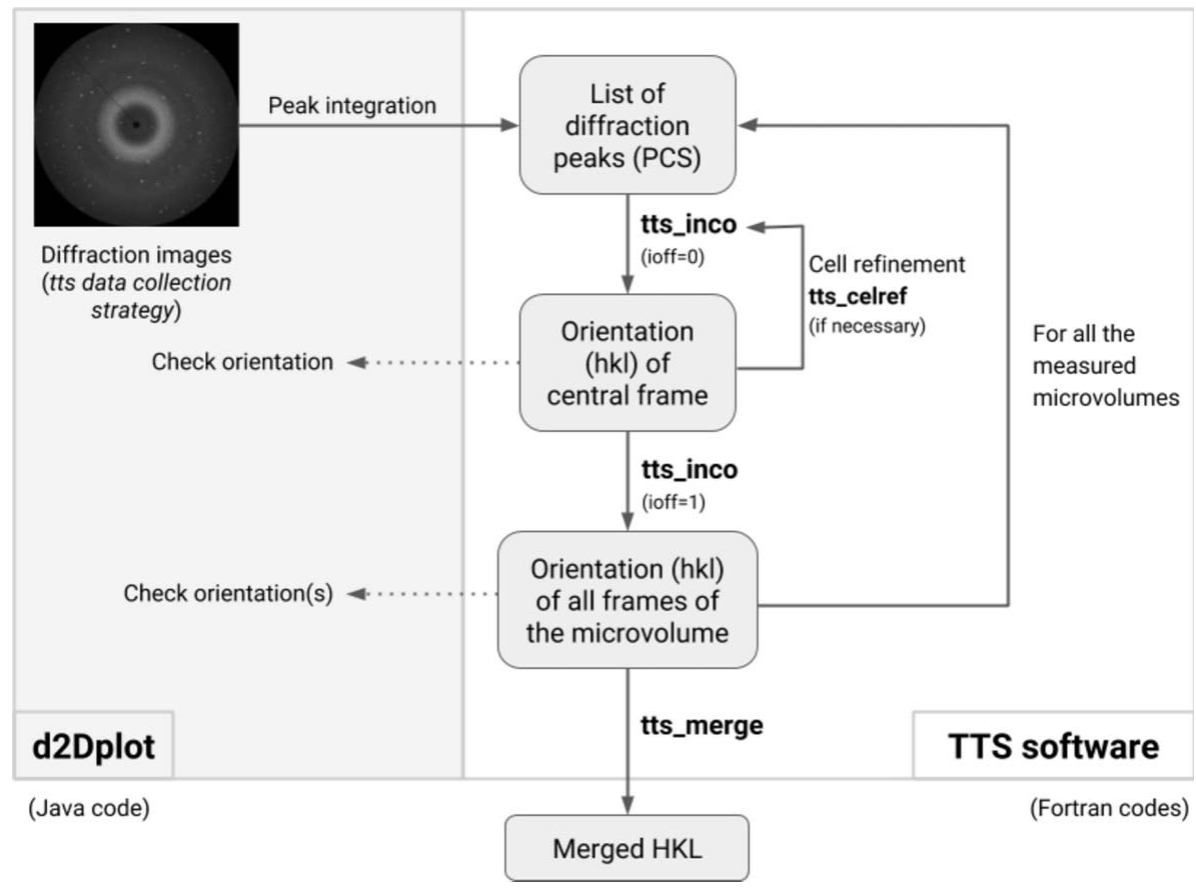

Figure 3

Typical $d 2$ Dplot and tts_software workflow, showing their complementarity. ground calculation is used (available as an option) then $I_{\text {mean }}$ and $\sigma_{I}$ refer to the background image estimated by the $a v s q$ method defined above (with $N=3$ ).

(2) That the eight neighbouring pixels have lower or equal intensities.

(3) That in a surrounding $5 \times 5$ grid pixel zone the number of pixels with intensity greater than $I_{\text {mean }}$ is higher than the minimum required number of pixels to consider it a peak (minpix).

Pixels surviving these tests provide a list of candidate peaks which are further processed as follows:

(4) Saturated peaks are labelled and the centroid is found when the plateau is spanned by more than one pixel.

(5) Close peaks are merged. The option peak merge zone defines the radius (in pixels) of the circular zone around each candidate peak. Peaks inside are merged and the final peak position is found by intensity weighting [Fig. 4(b)].

(6) Additional peak merging is performed along the azimuthal direction of each peak (double of the peak merge zone value) to minimize mosaicity effects.

The resulting diffraction peaks are integrated $\left(Y_{\text {sum }}\right)$ by adding the intensity of all the pixels inside an arc-shaped zone containing the peak and subtracting the background intensity of the zone. The zone dimension can be defined manually or automatically by checking the intensity variation in the radial and azimuthal directions from the peak centroid. At the end of the process, the peak search table contains the following information:

(i) Peak position $\left(x_{\text {pix }}, y_{\text {pix }}\right)$.

(ii) Distance in pixels from the beam centre to the peak (radius).

(iii) Maximum and mean intensity of the peak $\left(Y_{\text {max }}, Y_{\text {mean }}\right)$.

(iv) Intensity of the peak corrected for Lorentz and polarization effects according to

$$
\left|F_{H}\right|^{2}=Y_{\text {sum }} L^{-1} P^{-1},
$$

where

$$
\begin{aligned}
L^{-1} & =2 \sin \theta\left(\sin ^{2} \varphi-\sin ^{2} \theta\right)^{-1 / 2}, \\
P^{-1} & =\left(\cos ^{2} \psi+\sin ^{2} \psi \cos ^{2} 2 \theta\right)^{-1},
\end{aligned}
$$

with $\varphi$ being the angle between the axis of rotation and the reflecting plane, and with $\psi$ being the angle between the normal to the polarization plane and the vector going from the centre of the $2 \mathrm{D}$ detector to the $\left(x_{\text {pix }}, y_{\text {pix }}\right)$ pixel (Lipson et al., 2004). The associated standard deviation $\left(s_{F H 2}\right)$ is also given.

(v) Intensity of the background $\left(Y_{\mathrm{bkg}}\right)$ and the associated standard deviation $\left(s_{Y b k g}\right)$. 
(vi) Total number of pixels in the integration zone $\left(N_{\text {pix }}\right)$ and the number of saturated pixels (satur).

(vii) Radial width in pixels ( RadWth) and azimuthal aperture in degrees (AzimDeg) of the integration zone.

(viii) $d$ spacing of the peak in $\AA(d s p)$.

(ix) $p=\pi\left(Y_{\max } / Y_{\text {sum }}\right)^{2 / 3}$.

(x) Number of overlapping spots in the peak (swarm) and a flag to indicate if the peak integration zone is touching an excluded zone (nearMsk).

Fig. 4(a) shows the peak search result on a diffraction image of a polished thin section of a garnet (grossular) specimen mounted on a glass substrate. Diffraction data were collected at the microdiffraction/high-pressure endstation of the MSPD beamline at the ALBA Synchrotron (Fauth et al., 2013) with a Rayonix SX165 CCD detector (round active area of $165 \mathrm{~mm}$ diameter, frame size $2048 \times 2048$ pixels, $79 \mu \mathrm{m}$ pixel size, dynamic range $16 \mathrm{bit}$ ) at $184 \mathrm{~mm}$ of the sample using an energy of $29.2 \mathrm{keV}(0.4246 \AA)$ and a beam size of $15 \times 15 \mu \mathrm{m}$. Five frames were collected with $\Delta \varphi=7.5^{\circ}$ and an offset range -15 to $15^{\circ}$.

The results of the peak integration can be exported as a PCS file for the tts_software or as a text file. Batch integration of a set of images is also implemented.

3.2.2. tts software module. This module is a graphical interface for the tts_software to assist in the tts- $\mu$ XRD data

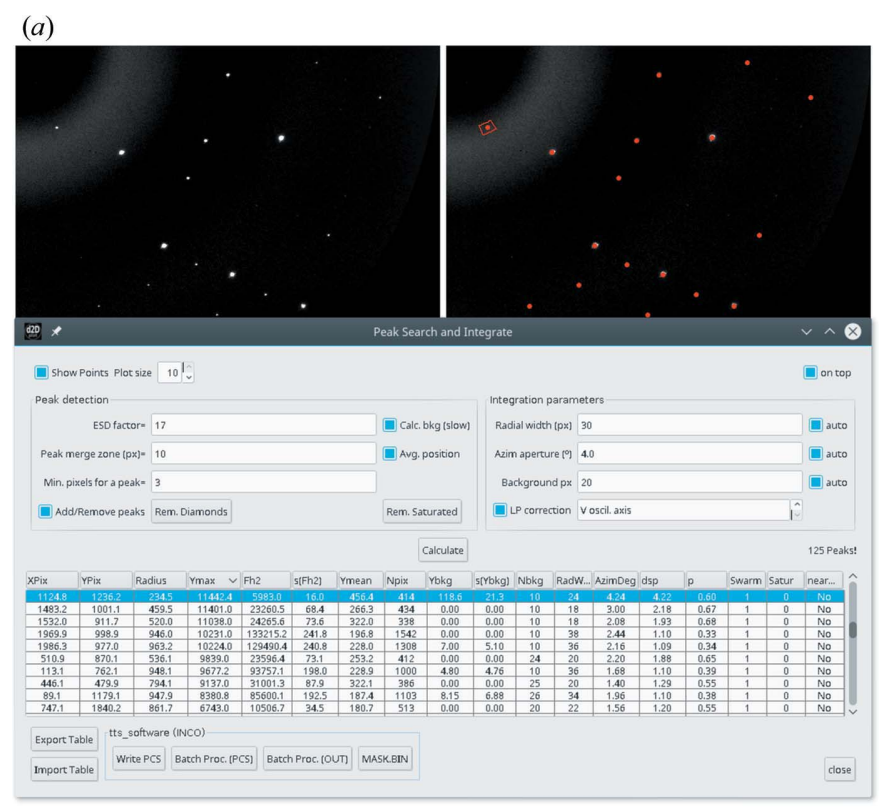

(b)

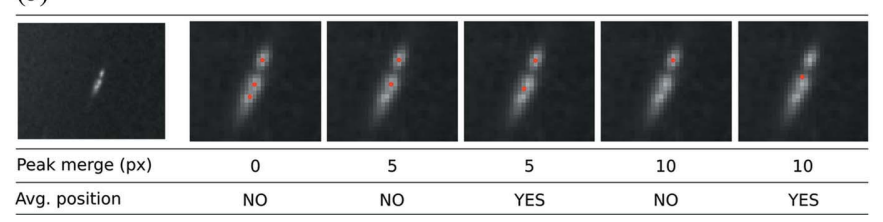

Figure 4

Peak identification module of d2Dplot. (a) Garnet 2DXRD after the peak search. The table contains information on the found peaks (red dots in the image showing the integration area for the selected ones). (b) Peak position assignment according to the peak merge zone and the intensity weighting. analysis. The tts_software must be downloaded separately (https://departments.icmab.es/crystallography/software) and then the executables path set in d2Dplot. The graphical interface helps in all the steps of the workflow (Fig. 3) by preparing the input files, launching the programs (tts_inco, tts_merge and tts_celref) and displaying the solutions at the different steps. For each orientation solution, the calculated reflection positions are displayed on the image so that the correct one (or several if there is more than one grain) can be easily identified (Fig. 5).

3.2.3. Examples of application. In addition to the examples and citations already mentioned in Section 1, there are other interesting applications showing the combined use of $d 2 \mathrm{Dplot}$ and $t$ s_software. Recently, the intensities of two different domains of the mineral dickinsonite contained in a single set of images [Fig. 6(a)] have been used to refine the crystal structure (article in preparation). A step-by-step tutorial for this example can be found in the user guide of the program. Another application under study is the crystal structure refinement of $\gamma$-trimesic acid at high pressure inside a diamond anvil cell. $\gamma$-Trimesic acid (orthorhombic, I222) is a flexible porous material (Sanchez-Sala et al., 2018) and the structural evolution with pressure has been followed by applying the tts- $\mu$ XRD methodology [Fig. 6(b)]. When the pressure is increased, the cell parameters and crystal orientation from the previous studied pressure $(1.3 \mathrm{GPa})$ are used as starting values for the orientation search and subsequent unit-cell refinement to match the experimental data at the current pressure $(1.8 \mathrm{GPa})$.

\subsection{User-created compound database and phase identifica- tion}

$d 2$ Dplot uses the $d$-spacing values of the compounds included in a database created by the user (UDB) to display the corresponding Debye rings on the current image by taking into account the instrumental parameters. The crystallographic
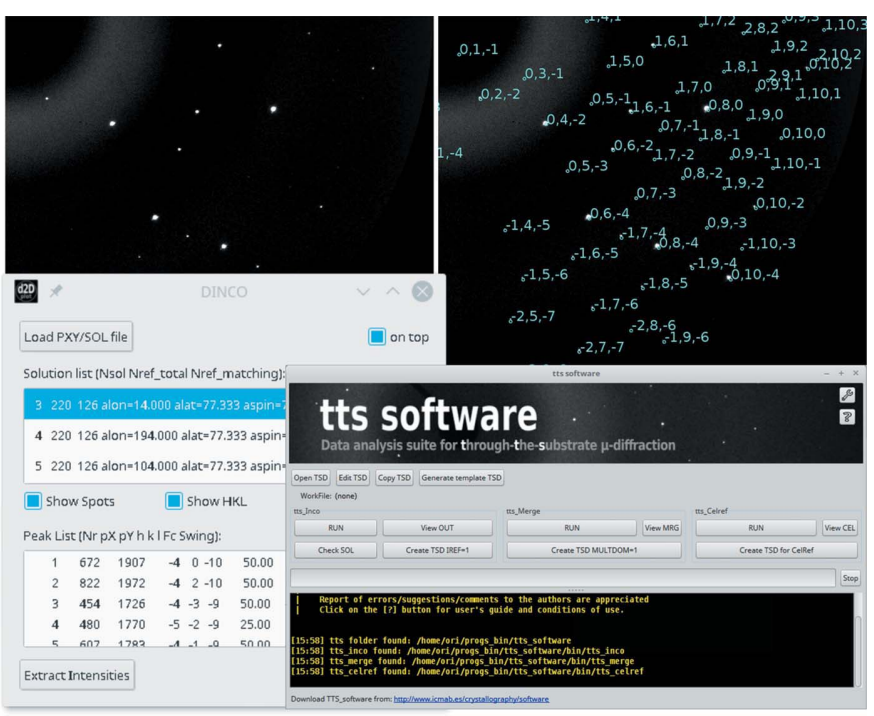

Figure 5

Solution of the orientation search from tts_inco displayed with $d 2$ Dplot. The corresponding $h k l$ indices of the reflections can also be shown. 
(a)

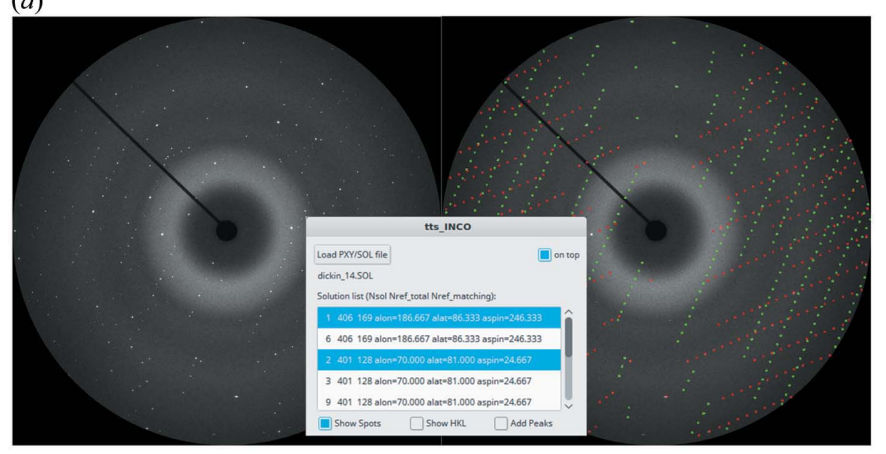

(b)

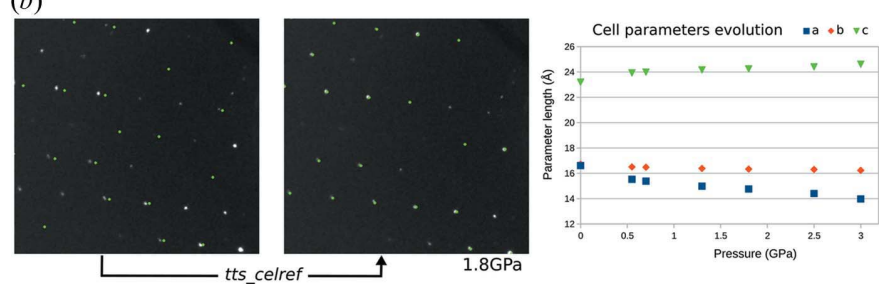

Figure 6

d2Dplot and tts_software applications. (a) Orientation results for two different domains of the mineral dickinsonite contained in the same microvolume. (b) Evolution of the $\gamma$-trimesic acid cell parameters as a function of pressure. Cell parameters are refined to match the new reflection positions when the pressure is increased. The green dots show the expected reflection positions before and after refining the unit cell.

information is stored in a plain-text format file to allow easy reading and modification. It consists of the list of $d$-spacing positions either entered as a file or generated from the corresponding CIF (Hall et al., 1991). In the latter case, intensities are also calculated and stored. Selection of the displayed compound and the operations involving the UDB are performed from the Compound DB window (Fig. 7). Phase identification from the peak positions is carried out considering the current selected reflections (left mouse button on the frame to select a reflection) and all the compounds in the database, showing those which better match the entered $d$-spacing values.

In the course of a tts- $\mu$ XRD experiment, it often occurs that the number of possible phases in a thin section is rather small. In such cases it is convenient to have direct access from the main window (via a Quicklist) to this small subset of phases in the UDB. Selection of a compound through the Quicklist option quickens the visual control of the compound matching, since opening of the UDB window is not necessary.

\subsection{Command-line mode}

The program can be launched from the command line giving the image file path as an argument to open it directly. In addition, instrumental parameters calibration and conversion of 2DXRD data to a $1 \mathrm{D}$ pattern can be launched from the command line without displaying the graphical interface. This is accomplished by entering -macro as the first argument followed by additional arguments depending on the operations performed. This mode is intended to be used for pipelines of operations on images, for example to automatically

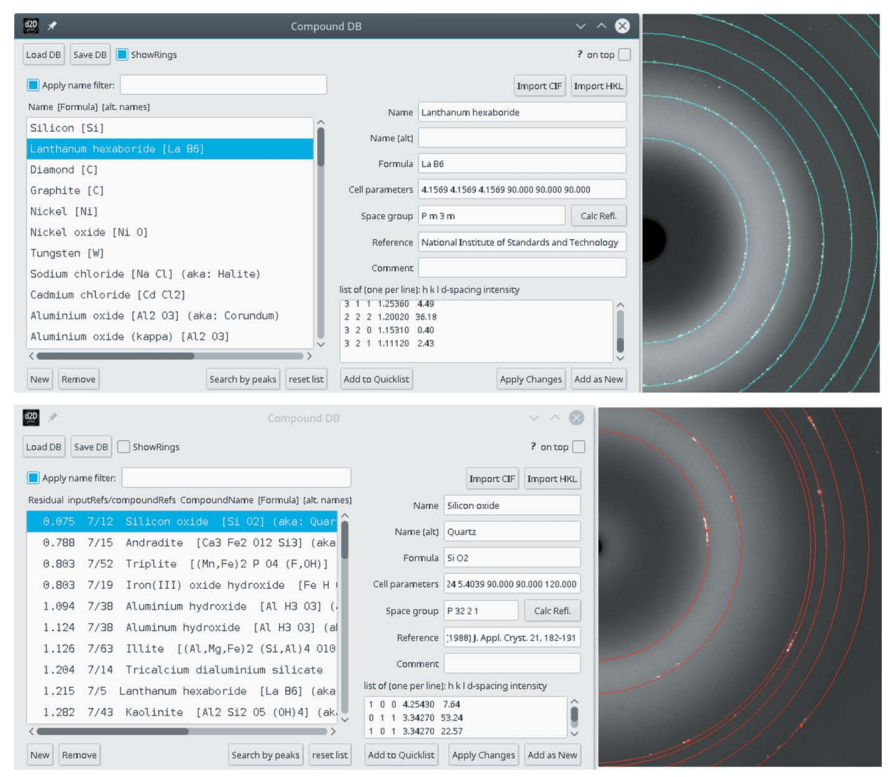

Figure 7

Database window showing (top) the list of compounds in the database with calculated rings displayed on the 2DXRD frame for the selected compound, and (bottom) the ranked list of matching compounds after the peak-based search.

generate the $1 \mathrm{D}$ pattern from 2DXRD data after collection with the $2 \mathrm{D}$ detector.

\section{Conclusions}

$d 2$ Dplot acts as the graphical interface of the tts_software. In the analysis of crystalline microvolumes with the tts- $\mu \mathrm{XRD}$ methodology, it simplifies its application by guiding and helping users through the full workflow. $d 2$ Dplot provides a complete set of tools for the visualization and processing of 2DXRD data, especially for applications where the detailed inspection of diffraction frames is important. It also serves as a tool during the data collection stage, offering quick phase identification or batch processing capabilities. The combined use of $d 2 D$ plot and tts_software has been successfully applied to the in situ characterization of polished thin sections in complex mineralogical and cultural heritage problems.

\section{Acknowledgements}

OV thanks the ALBA Synchrotron and the MSPD beamline, in particular Dr Francois Fauth for the support and feedback regarding this software.

\section{Funding information}

Financial support by projects MAT2015-67593-P and 'Severo Ochoa' (SEV-2015-0496) of MINECO and FEDER is acknowledged.

\section{References}

Agilent Technologies (2014). CrysAlis PRO. Agilent Technologies Ltd, Yarnton, UK. 
Arndt, U. W. \& Wonacott, A. J. (1977). The Rotation Method in Crystallography: Data Collection from Macromolecular Crystals. Amsterdam: North-Holland Publishing Co.

Arvai, A. J. (1994). Adxv - a Program to Display X-ray Diffraction Images, https://www.scripps.edu/tainer/arvai/adxv.html.

Arvai, A. J. \& Nielsen, C. (1983). ADSC Quantum-210 ADX. Area Detector System Corporation, Poway, CA, USA.

Ashiotis, G., Deschildre, A., Nawaz, Z., Wright, J. P., Karkoulis, D., Picca, F. E. \& Kieffer, J. (2015). J. Appl. Cryst. 48, 510-519.

Battye, T. G. G., Kontogiannis, L., Johnson, O., Powell, H. R. \& Leslie, A. G. W. (2011). Acta Cryst. D67, 271-281.

Bernstein, H. J. \& Hammersley, A. P. (2005). International Tables for Crystallography, Vol. G, Definition and Exchange of Crystallographic Data, edited by S. R. Hall \& B. McMahon, pp. 37-43. Dordrecht: Springer.

Brückner, S. (2000). J. Appl. Cryst. 33, 977-979.

Bruker (1999). General Area Detector Diffraction System (GADDS). User Manual. Bruker AXS, Madison, WI, USA.

Faber, J. \& Fawcett, T. (2002). Acta Cryst. B58, 325-332.

Fauth, F., Peral, I., Popescu, C. \& Knapp, M. (2013). Powder Diffr. 28, S360-S370.

Hall, S. R., Allen, F. H. \& Brown, I. D. (1991). Acta Cryst. A47, 655685.

Hammersley, A. P. (1997). FIT2D: an Introduction and Overview. ESRF Internal Report ESRF97HA02T. European Synchrotron Research Facility, Grenoble, France.

Hammersley, A. P. (2016). J. Appl. Cryst. 49, 646-652.

Hammersley, A. P., Svensson, S. O. \& Thompson, A. (1994). Nucl. Instrum. Methods Phys. Res. A, 346, 312-321.

Hart, M. L., Drakopoulos, M., Reinhard, C. \& Connolley, T. (2013). J. Appl. Cryst. 46, 1249-1260.

He, B. B. (2009). Two-Dimensional X-ray Diffraction. Hoboken: John Wiley \& Sons.
Hinrichsen, B., Dinnebier, R. E. \& Jansen, M. (2008). Powder Diffraction, pp. 414-438. Cambridge: Royal Society of Chemistry. Kabsch, W. (1988). J. Appl. Cryst. 21, 916-924.

Kieffer, J. \& Karkoulis, D. (2013). J. Phys. Conf. Ser. 425, 202012.

Lipson, H., Langford, J. I. \& Hu, H.-C. (2004). International Tables for Crystallography, Vol. C, Mathematical, Physical and Chemical Tables, edited by E. Prince, pp. 596-598. Dordrecht: Kluwer.

Maritan, L., Casas, L., Crespi, A., Gravagna, E., Rius, J., Vallcorba, O. \& Usai, D. (2018). Herit. Sci. 6, 74.

Maritan, L., Piovesan, R., Dalconi, M. C., Rius, J., Crespi, A., Vallcorba, O., Casas, L., Vidale, M. \& Olivieri, L. M. (2018). Minerals, 8, 200.

Rius, J., Vallcorba, O., Crespi, A. \& Colombo, F. (2017). Z. Kristallogr. Cryst. Mater. 232, 827-834.

Rius, J., Vallcorba, O. \& Frontera, C. (2017). TTS Software: Computer Software for Crystal Structure Analysis From tts Microdiffraction Data. User Manual. Institut de Ciencia de Materials de Barcelona, CSIC, Spain.

Rius, J., Vallcorba, O., Frontera, C., Peral, I., Crespi, A. \& Miravitlles, C. (2015). IUCrJ, 2, 452-463.

Sanchez-Sala, M., Vallcorba, O., Domingo, C. \& Ayllón, J. A. (2018). Cryst. Growth Des. 18, 6621-6626.

Schmalen, P., Plapper, P., Peral, I., Titov, I., Vallcorba, O. \& Rius, J. (2018). Procedia CIRP, 74, 27-32.

Schneider, C. A., Rasband, W. S. \& Eliceiri, K. W. (2012). Nat. Methods, 9, 671-675.

Toby, B. H. \& Von Dreele, R. B. (2013). J. Appl. Cryst. 46, 544-549. Vallcorba, O., Casas, L., Colombo, F., Frontera, C. \& Rius, J. (2017). Eur. J. Mineral. 29, 287-293.

Winter, G., Waterman, D. G., Parkhurst, J. M., Brewster, A. S., Gildea, R. J., Gerstel, M., Fuentes-Montero, L., Vollmar, M., MichelsClark, T., Young, I. D., Sauter, N. K. \& Evans, G. (2018). Acta Cryst. D74, 85-97. 